\title{
Comparison of capecitabine-based regimens with platinum-based regimens in Chinese triple-negative breast cancer patients with liver metastasis
}

\author{
Yimeng Chen ${ }^{1}$, Yin Guan ${ }^{2}$, Jiayu Wang ${ }^{1}$, Fei Ma ${ }^{1}$, Yang Luo ${ }^{1}$, Shanshan Chen ${ }^{1}$, Pin Zhang ${ }^{1}$, Qing Li $^{1}$, \\ Ruigang Cai ${ }^{1,3}$, Qiao $\mathrm{Li}^{1}$, Hongnan $\mathrm{Mo}^{1}$, Ying Fan ${ }^{1}$, Weihong $\mathrm{Zhao}^{4}$, Binghe $\mathrm{Xu}^{1}$ \\ ${ }^{1}$ Department of Medical Oncology, National Cancer Center/National Clinical Research Center for Cancer/Cancer Hospital, Chinese Academy \\ of Medical Sciences and Peking Union Medical College, Beijing, China; ${ }^{2}$ Department of Medical Oncology, Beijing Chao-Yang Hospital, Beijing, \\ China; ${ }^{3}$ Department of Medical Oncology, Beijing Sanhuan Cancer Hospital, Beijing, China; ${ }^{4}$ Department of Medical Oncology, Chinese PLA \\ General Hospital, Beijing, China \\ Contributions: (I) Conception and design: Y Fan; (II) Administrative support: B Xu, W Zhao, Y Guan; (III) Provision of study materials or patients: \\ J Wang, F Ma, Y Luo, S Chen, P Zhang, Q Li, R Cai, Q Li; (IV) Collection and assembly of data: Y Chen, Y Guan, H Mo; (V) Data analysis and \\ interpretation: Y Chen, H Mo; (VI) Manuscript writing: All authors; (VII) Final approval of manuscript: All authors. \\ Correspondence to: Ying Fan. Department of Medical Oncology, National Cancer Center/National Clinical Research Center for Cancer/Cancer \\ Hospital, Chinese Academy of Medical Sciences and Peking Union Medical College, Beijing 100021, China. Email: fanyingfy@medmail.com.cn; \\ Weihong Zhao. Department of Medical Oncology, Chinese PLA General Hospital, Beijing 100853, China. Email: zhaowh0818@163.com; Binghe \\ Xu. Department of Medical Oncology, National Cancer Center/National Clinical Research Center for Cancer/Cancer Hospital, Chinese Academy \\ of Medical Sciences and Peking Union Medical College, Beijing, China. Email: xubinghebm@163.com.
}

Background: Capecitabine-based chemotherapy (CBC) presents potential value in patients with liver metastasis; platinum-based chemotherapy (PBC) has shown promising benefit in patients with triple-negative breast cancer (TNBC). For TNBC patients with liver metastasis, which treatment strategy is better remains to be further studied. The aim of this study was to report the first real-world data evaluating the efficacy and safety of PBC versus CBC in the first-line treatment in Chinese TNBC patients with liver metastasis.

Methods: TNBC patients with liver metastasis pretreated with anthracyclines/taxanes in 4 institutions of China between January 2010 and December 2019 were included. Objective response rate (ORR), overall survival, treatment pattern, and toxicity profile were assessed between $\mathrm{PBC}$ and $\mathrm{CBC}$ groups.

Results: A total of 59 TNBC patients with liver metastasis were identified. Among these, 33 were treated with $\mathrm{PBC}$ and 26 were treated with $\mathrm{CBC}$. The ORR was higher in the $\mathrm{CBC}$ group than in the PBC group (57.7\% versus $30.3 \%, \mathrm{P}=0.035$ ). Median overall survival was also greatly improved (19.2 versus 14.4 months, $\mathrm{P}=0.041$ ). Docetaxel/cisplatin was more likely to be used for $\mathrm{PBC}$, and paclitaxel/capecitabine was the main regimen for CBC. Multivariable Cox regression analysis indicated that CBC was an independent predictor for overall survival after adjustment for baseline factors including age, tumor size, nodal status, prior anthracyclines/taxanes use, and tumor grade (odds ratio $=0.51 ; 95 \%$ confidence interval, $0.27-0.98$; $\mathrm{P}=0.042$ ). Adverse events were not different except gastrointestinal tract toxicities, hand-foot syndrome and hematologic toxicity.

Conclusions: For TNBC patients with liver metastasis, capecitabin-based chemotherapy might be more suitable than the platinum-based regimen in the first-line treatment, as measured by objective response rate and overall survival. Further large-scale studies are warranted.

Keywords: Capecitabine; platinum; triple-negative breast cancer (TNBC); liver metastasis

Submitted Jun 09, 2020. Accepted for publication Oct 30, 2020.

doi: $10.21037 /$ atm-20-4590

View this article at: http://dx.doi.org/10.21037/atm-20-4590

(c) Annals of Translational Medicine. All rights reserved. 


\section{Introduction}

Triple-negative breast cancer (TNBC) refers to the absence of estrogen receptor (ER), progesterone receptor $(\mathrm{PgR})$, and human epidermal growth factor receptor-2 (HER-2). It is a specific subtype of breast cancer accounting for $15-$ $20 \%$ of all breast cancers (1). TNBC presents a trend of early visceral metastasis, and has poorer prognosis (2). Among patients with metastatic breast cancer (MBC), approximately half will develop liver metastases (LM), and $12 \%$ of patients develop metastasis of primary liver cancer (3), leading to liver dysfunction and poor survival (4).

Instead of available target agents, chemotherapy represents the mainstay systemic treatment for metastasis TNBC (mTNBC) (5). Anthracyclines and taxanes are fundamental regimens with proven efficacy in every stage of breast cancer (6). However, for patients with anthracycline/ taxanes-pretreated breast cancers, there are no standard regimens currently (7).

For patients with mTNBC, platinum-based chemotherapy (PBC) has shown promising results in increasing preclinical and clinical trials. Platinum can lead to DNA damage, and TNBC is more sensitive to these agents compared to other subtypes of breast cancers. The benefit of platinum regimens was confirmed by several II or III clinical trials (8-10).

For patients with LM, capecitabine-based chemotherapy (CBC) seems to show potential value. Capecitabine is among the drugs of first choice for breast cancer patients resistant to anthracycline or taxane (11). It is activated in the liver and further forms 5-FU in the tumor tissue (12-14), suggesting higher concentration in the liver and the potential benefit for patients with liver metastases (LM).

However, for mTNBC patients with LM, which agents are more suitable is uncertain. In this study, we present the result of the comparison of the $\mathrm{PBC}$ and $\mathrm{CBC}$ in patients with mTNBC-LM.

We present the following article in accordance with the STROBE reporting checklist (available at http://dx.doi. org/10.21037/atm-20-4590).

\section{Methods}

\section{Patients and data collection}

In this study, we retrospectively compared the efficacy and toxicity of CBC and PBC in mTNBC patients with LM. We reviewed the electronic medical records of patients with mTNBC who received systematical chemotherapy at four cancer centers in China (National Cancer Center, Chinese PLA General Hospital, Beijing Chaoyang Hospital, Beijing Sanhuan Cancer Hospital) between January 2010 and December 2019.

Inclusion criteria were as follows for eligible patients: (I) immunohistochemical (IHC) staining method was applied to determine the ER/PgR/HER-2status. Triple negativity breast cancer was defined as the deficiency of expression of ER, PgR, and HER-2. "ER/PgR negative" were defined when less than $1 \%$ positive tumor cells were detected with nuclear staining by IHC according to the guidelines of new College of American Pathologists. HER-2 status was evaluated by IHC and fluorescence in situ hybridization (FISH). "HER-2 negative" was defined as IHC scoring 0 or $1+$ or FISH nonamplied according to the American Society of Clinical Oncology (ASCO) guidelines. (II) Breast cancers patients had initial isolated liver metastasis. (III) Patients received $\mathrm{PBC}$ or $\mathrm{CBC}$ as the first-line treatment. (IV) Patients had completed treatment records and followup information. Finally, 59 eligible TNBC patients with LM were included in this study (Figure 1). Initial LM was defined as LM that occurred as the first evidence of metastasis, and isolated LM was characterized by the absence of extrahepatic metastasis.

Patients were further allocated into two groups by their regimens in their salvage chemotherapies: the $\mathrm{PBC}$ group and $\mathrm{CBC}$ group. $\mathrm{PBC}$ referred to chemotherapy that included cisplatin or carboplatin alone or in combination with another regimen. CBC was defined as chemotherapy that included capecitabine alone or in combination with other regimens. Clinical data were collected and analyzed, including demographic characteristics, disease stage at diagnosis, treatment regimens, response to treatment, adverse events, and overall survival.

The study was conducted in accordance with the Declaration of Helsinki (as revised in 2013). It was approved by the Ethics Committee of National Cancer Center/ National Clinical Research Center for Cancer/Cancer Hospital, Chinese Academy of Medical Sciences and Peking Union Medical College (No.: 15-115/1042). Because of the retrospective nature of the research, the requirement for informed consent was waived.

\section{Response assessment and follow-up}

Tumor response was evaluated according to the response evaluation criteria in solid tumors (RECIST) 1.1 guideline, which was classified into four categories: complete response 


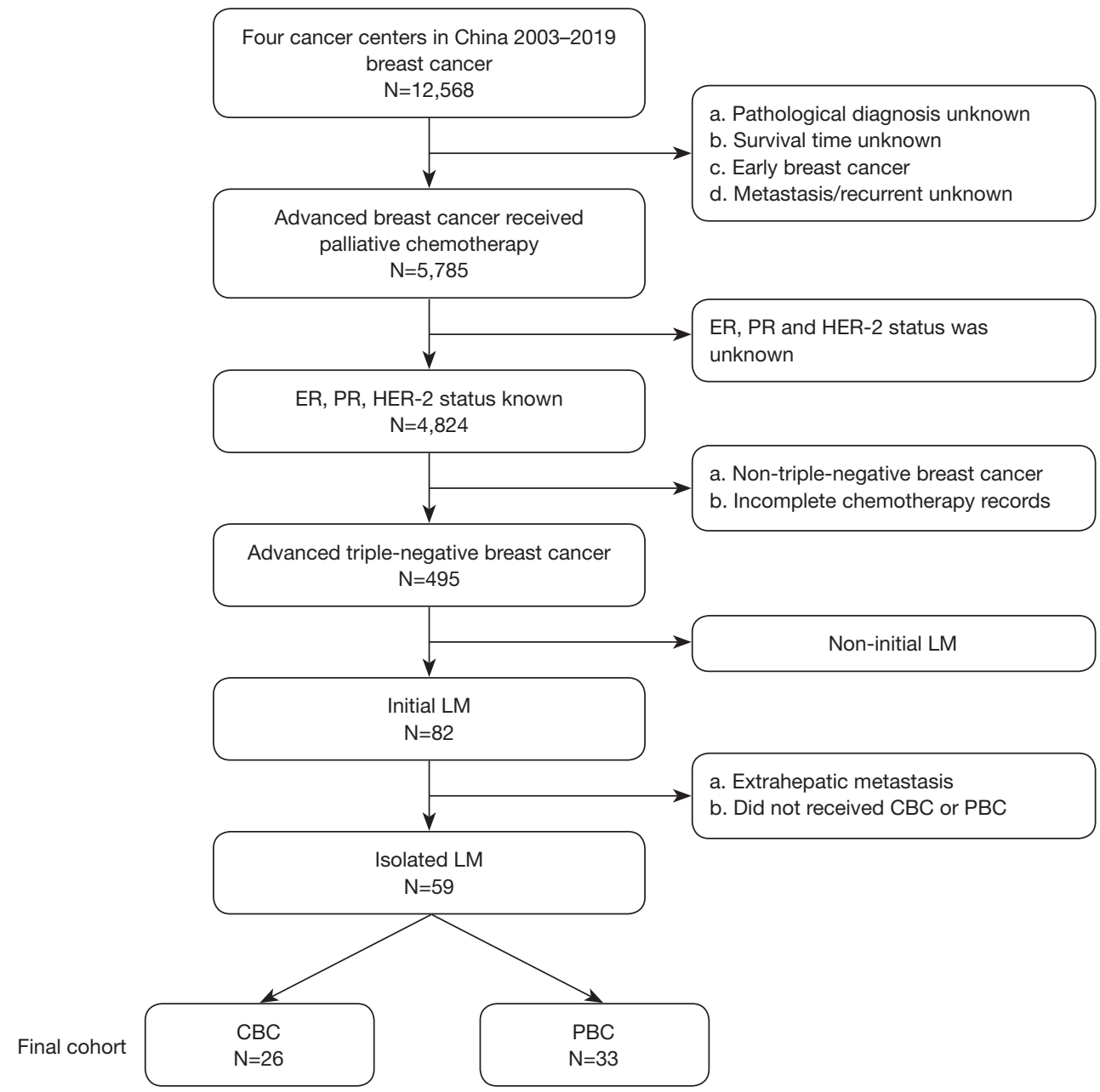

Figure 1 Flow diagram of patient selection.

(CR), partial response (PR), stable disease (SD), and progressive disease (PD). Tumor response to treatment was assessed every 2 cycles during chemotherapy and then every 3 months after chemotherapy. The efficacy of chemotherapy was evaluated in terms of objective response rate (ORR) and overall survival (OS). Adverse events were evaluated based on the Common Terminology Criteria for Adverse Events (CTCAE) 4.03.

\section{Statistical analysis}

OS was defined as the interval from the time of diagnosis of the liver metastasis to the time of death or until the date of the last follow-up visit. OS was computed according to the Kaplan-Meier method, and compared by the log-rank test. Multivariate survival analysis was performed according to the Cox proportional hazards model. Statistical analysis was performed via SPSS software version 22.0. A P value less than 0.05 was considered to be statistically significant.

\section{Results}

\section{Characteristics of patients}

We identified 12,568 patients with breast cancer who had available treatment data from 2003 to 2019. Of these patients, 5,785 received palliative chemotherapy for the treatment of metastatic or recurrent breast cancer at four major cancer centers in China (National Cancer Center, Chinese PLA General Hospital, Beijing Chaoyang Hospital, Beijing Sanhuan Cancer Hospital). Of the 5,785 patients, 961 patients with $\mathrm{MBC}$ were excluded due to their unknown ER/PgR/HER2 status. Among the remaining patients, 
4,329 patients who were not TNBCs or had incomplete chemotherapy records were excluded from this study. Eighty-two patients were diagnosed with initial LM on the basis of abdominal computed tomographic scans or magnetic resonance imaging scans. LM was confirmed pathologically if necessary. Among these, patients who were not isolated $\mathrm{LM}$ and did not receive $\mathrm{PBC}$ or CBC as the first-line treatment were excluded. Finally, 59 patients were confirmed eligible and included for the final analysis (Figure 1).

Patient demographics at baseline are presented in Table 1. In total, 59 eligible patients were included in this study between January 2003 and December 2019. Of these patients, 33 patients were treated with $\mathrm{PBC}$, and 26 were treated with $\mathrm{CBC}$. The median age at diagnosis of the two cohorts was 48 [32-73] years and 52 [28-73] years, respectively. Premenopausal patients were dominant in the two groups. All patients had failed treatment with anthracyclines and taxanes. The majority of patients $(66.7 \%$ for PBC; $53.8 \%$ for $\mathrm{CBC}$ ) presented with pathological $\mathrm{T} 2$ tumors, and more than half $(54.6 \%$ for PBC; $61.6 \%$ for docetaxel) had N0/1 axillary nodes. The median Ki67 expression was $40 \%$ in $\mathrm{PBC}$ and $50 \%$ in CBC. Diseasefree interval (DFI) was 21.7 months in patients with $\mathrm{PBC}$, similar with that in $\mathrm{CBC}(\mathrm{P}=0.56)$. Overall, the two groups were well balanced in baseline characteristics.

\section{Chemotherapy regimens}

In the PBC group, 19 (57.6\%) patients received platinum agents (carboplatin or cisplatin) combined with taxanes (TP), $9(27.2 \%)$ patients were treated with gemcitabine+platinum (GP), and $5(15.1 \%)$ were treated with vinorelbine+platinum (NP). The main strategies of CBC were taxane-containing regimens (TX, $\mathrm{n}=18,65.3 \%$ ), followed by vinorelbine/capecitabine combinations (NX, $\mathrm{n}=6,23.1 \%)$, and capecitabine monotherapy (X, n=1, $3.8 \%)$. Carboplatin was administered at the area under the curve equal to $5(\mathrm{AUC}=5)$ on the first day every 3 weeks. Cisplatin was given at a dose of $25 \mathrm{mg} / \mathrm{m}^{2} /$ day on the first 3 days every 3 weeks. Capecitabine was delivered at a dose of $1,250 \mathrm{mg} / \mathrm{m} 2$ for the first 2 weeks every 21 days. The treatment strategies in the two cohorts are listed in Table 2.

\section{Response and survival}

The overall response rate (ORR) was 30.3\% (10/33) in the PBC group, including 1 complete response (CR, 3.1\%) and 9 partial response (PR, 27.3\%); 15(45.5\%) patients had stable disease (SD) and 8 (24.2\%) experienced progressive disease (PD) during the systematic treatment. In the CBC cohort, ORR was $57.7 \%$ (15/26), consisting of 1 (3.8\%) CR, 14 (53.8\%) PR, 8 (30.8\%) SD, and 3 (11.5\%) PD. There was a significantly higher response rate in patients who received $\mathrm{CBC}(57.7 \%)$ than that in patients who received PBC (30.3\%, P=0.035, Table 3).

During the follow-up, 42 patients died and 17 patients remained alive. Median $\mathrm{OS}$ in the $\mathrm{PBC}$ group was 14.4 months (95\% CI, 9.9-18.9 months), which was statistically longer than that in the CBC group (19.2 months, 95\% CI, 10.7-27.7 months, $\mathrm{P}=0.041)$, as illustrated in Figure 2. Multivariate Cox proportional hazards analysis was performed to eliminate confounding variables and to clarify whether $\mathrm{CBC}$ alone conferred a survival benefit. Univariate analysis was performed to explore potential prognostic factors; all of the variables with $\mathrm{P}<0.1$ were included in the Cox multivariate analysis (Table 4). The results indicated that $\mathrm{CBC}$ remained an independent predictor for OS after adjustment for baseline factors including age, tumor size, nodal status, prior anthracycline/taxane use, and tumor grade (OR 0.51; 95\% CI, 0.27-0.98; $\mathrm{P}=0.042$, Table 4).

\section{Toxicity}

Major treatment-related adverse effects (TRAEs) are shown in Table 5, mainly including vomiting, neutropenia, leucopenia, hand-foot syndrome, hepatic abnormalities, and fatigue. During the treatment, $93.9 \%$ of patients treated with PBC had at least one TRAEs compared with $96.2 \%$ of those treated with CBC. Gastrointestinal tract adverse events occurred more frequently and were more severe with $\mathrm{PBC}$ than $\mathrm{CBC}$ at grade $1 / 2(\mathrm{P}=0.015)$ and at grade $3 / 4(\mathrm{P}=0.045)$. Hand-foot syndrome was more common in the CBP group especially at grade $3 / 4(37.2 \%$ vs. $18.0 \%$, $\mathrm{P}=0.037)$. Neutropenia $(75.8 \%$ vs. $38.5 \%, \mathrm{P}=0.004)$ and leucopenia $(78.8 \%$ vs. $46.2 \%, \mathrm{P}=0.009)$ at grade $1 / 2$ also occurred more frequently in the $\mathrm{PBC}$ group than in the CBC groups. Incidences of diarrhea, fatigue, and hepatic abnormalities were comparable between the two groups. There were no treatment-related deaths in either group. Generally, both treatment strategies were tolerated and quite manageable.

\section{Discussion}

Studies focused on mTNBC indicated that capecitabinebased regimens could prolong the survival time (15-17). 
Table 1 Baseline characteristics of patient with metastatic triple-negative breast cancer

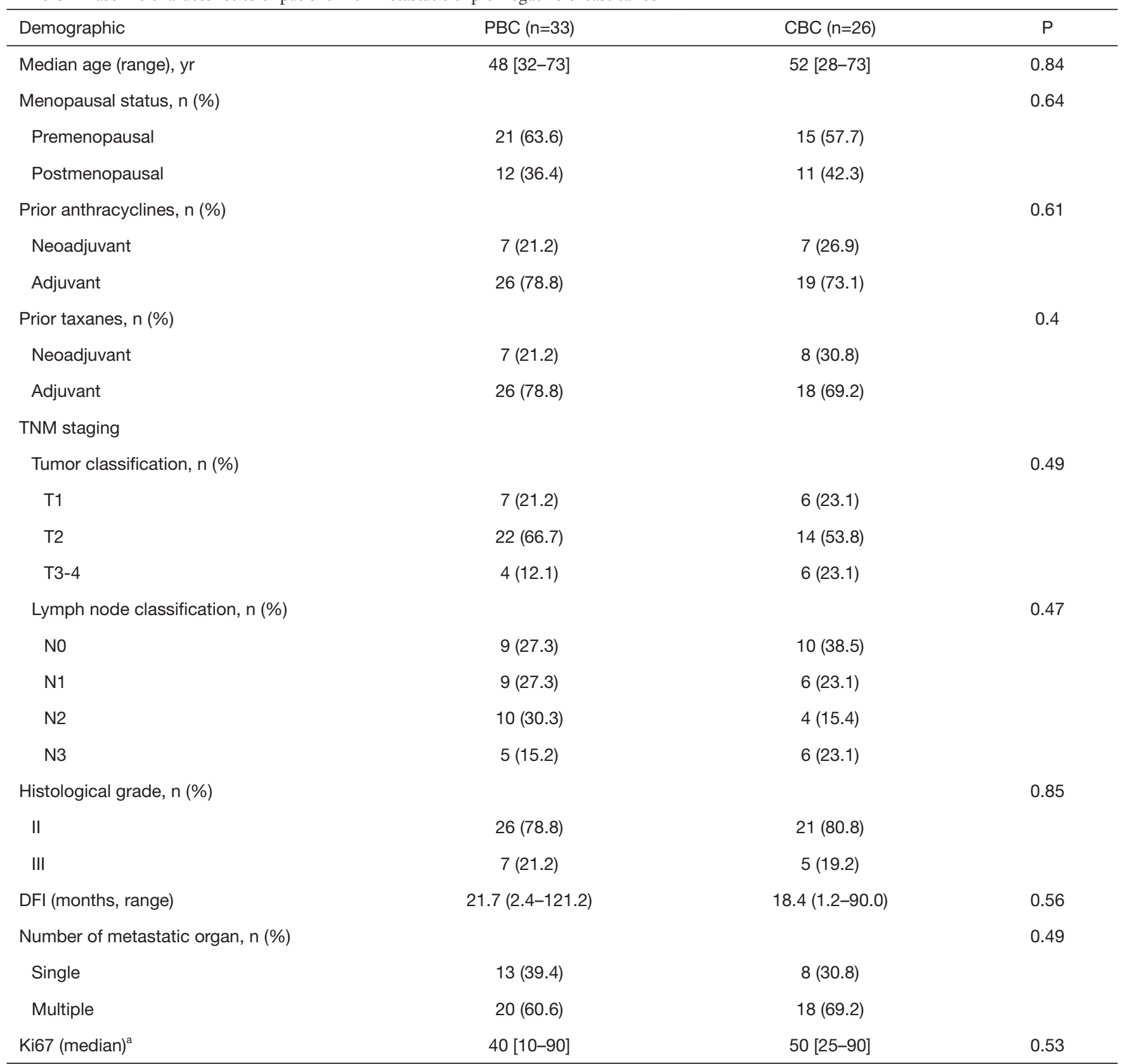

a, some of Ki67 index from local hospital were missing. CBC, carboplatin-based chemotherapy; DFI, disease-free interval defined as the time from operation to first relapse; PBC, platinum-based chemotherapy.

Most recent studies showed that PBC in TNBC patients has promising results in increasing preclinical and clinical trials. The platinum-based regimen has higher ORR and better survival than the platinum-free regimen (8-10). However, study focused on TNBC patients with LM is scarce.

With regard to the present study, we compared the efficacy and safety profile of capecitabine-based regimens with platinum-based regimens in TNBC patients with LM. The result demonstrated that the CBC group achieved higher ORR and longer OS than that in the PBC group, with tolerable adverse events except incidence of handfoot syndrome. To the best of our knowledge, this is the 
Table 2 Chemotherapy regimens

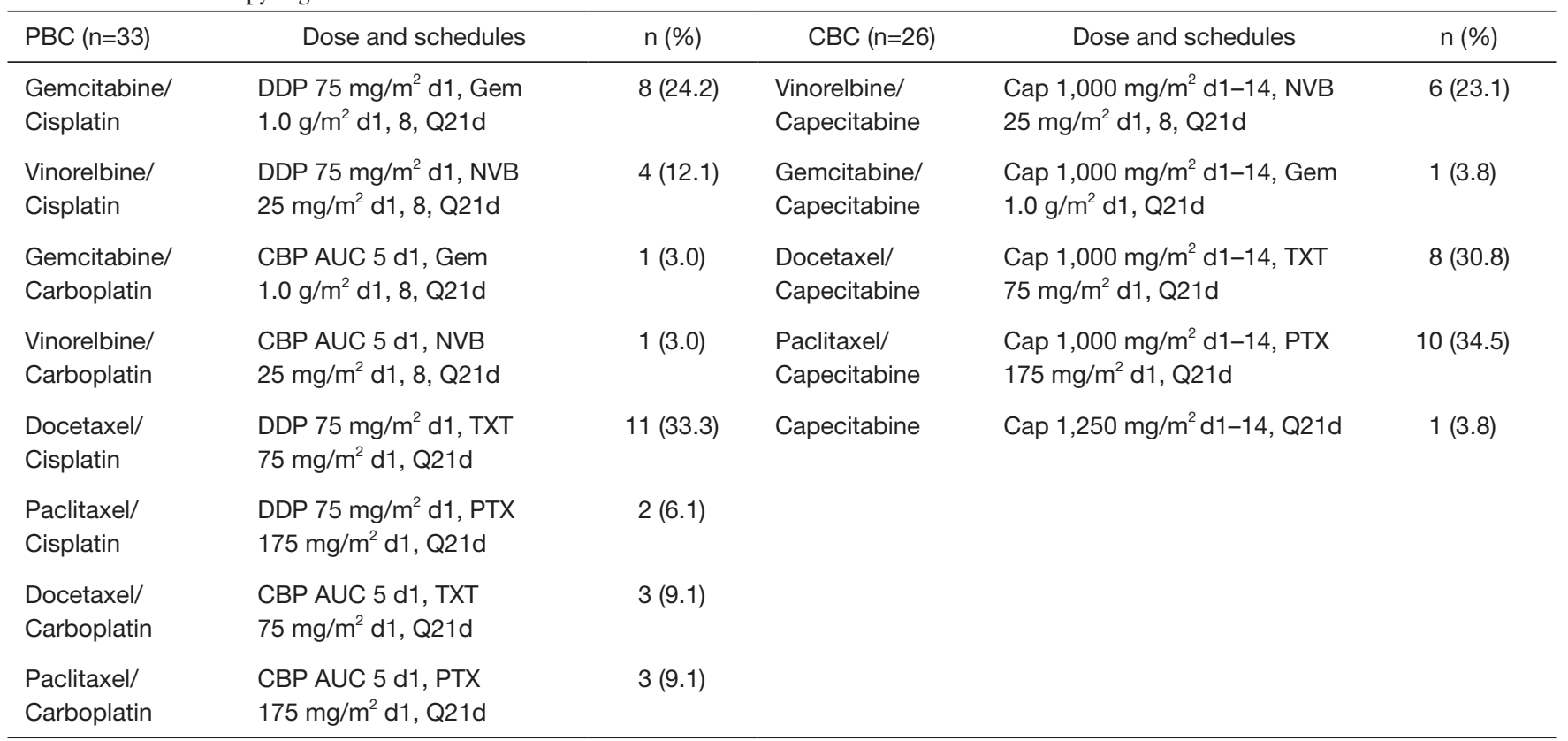

AUC, area under the curve; Cap, capecitabine; CBC, capecitabine-based chemotherapy; CBP, carboplatin; DDP, cisplatin; Gem, gemcitabine; NVB, vinorelbine; PTX, paclitaxel; PBC, platinum-based chemotherapy; TXT, docetaxel.

Table 3 Best tumor response to chemotherapy

\begin{tabular}{lcc}
\hline Tumor response & PBC $(\mathrm{n}=33, \%)$ & $\mathrm{CBC}(\mathrm{n}=26, \%)$ \\
\hline CR & $1(3.1)$ & $1(3.8)$ \\
PR & $9(27.3)$ & $14(53.8)$ \\
SD & $15(45.5)$ & $8(30.8)$ \\
PD & $8(24.2)$ & $3(11.5)$ \\
\hline
\end{tabular}

CBC, capecitabine-based chemotherapy; CR, complete response; PBC, platinum-based chemotherapy; PD, progression disease; PR, partial response; SD, stable disease.

first investigation to compare the role of $\mathrm{PBC}$ with $\mathrm{CBC}$ in TNBC patients with LM.

Our data showed that the ORR $(57.7 \%$ vs. $30.3 \%$, $\mathrm{P}=0.035)$ was higher in the $\mathrm{CBC}$ group, and the median OS (19.2 vs. 14.4 months, $\mathrm{P}=0.041)$ was greatly improved compared with the PBC group. These findings are also consist with most other studies supporting a statistically significant benefit on response rates and median OS from capecitabine in anthracycline- and taxane-pretreated MBC (15-17). Results from several meta-analysis showed that capecitabine could significantly improve the survival both in patients with early and advanced breast cancer $(18,19)$. Capecitabine might prolong OS in patients with ERnegative or HER2-negative breast cancer in the first-line treatment. Moreover, adjuvant capecitabine therapy showed effectiveness in triple-negative subgroup (19).

In our study, we found that capecitabine showed an active efficacy in a liver metastatic setting. This observation was in line with the previous studies (20-27). Findings from the study of colorectal cancer patients with LM indicated that the concentration of 5-FU (precursor of capecitabine) in liver metastasis tissue was higher than normal colorectal tissue and reached about $70 \%$ of the number in colorectal tumor tissue (20). Possible explanations might be the higher concentration of $5-\mathrm{Fu}$ in the liver $(21,22)$. The chemotherapy response may be influenced by the distribution of drugs and its concentration in tumor tissues. Capecitabine is an oral chemotherapy drug and is 


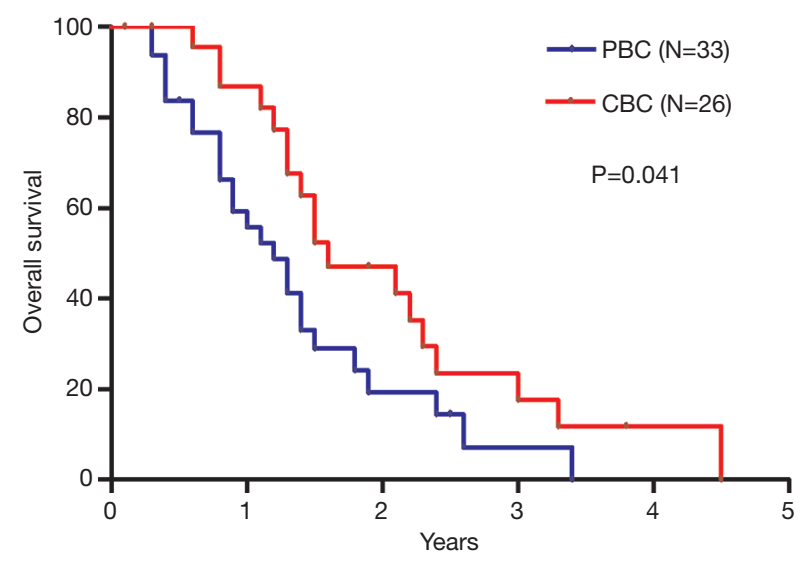

Figure 2 Kaplan-Meier curves of overall survival for patients treated with capecitabine-based chemotherapy $(\mathrm{N}=26)$ and platinum-based chemotherapy $(\mathrm{N}=33)$. enzymatically converted into $5-\mathrm{FU}$ in the liver and tumor tissues $(23,24)$. The activity of thymidine phosphorylase (TP) is much higher in normal liver tissues than that in other tissues (25). The benefit of capecitabine-based therapy (CBT) is significantly affected by the activity of TP (26). Another possible explanation might be the maintenance chemotherapy of capecitabine (27). Maintenance chemotherapy refers to the continuous treatment of part of the primary agents in patients after the initial standard treatment, which can significantly prolong the survival time. Capecitabine-based regimens usually ends with the maintenance of capecitabine monotherapy $(28,29)$.

Generally, both strategies were well tolerated and manageable. In the CBC group, the most frequent adverse effects were neutropenia, leucopenia and hand-

Table 4 Univariate and multivariate Cox regression analyses

\begin{tabular}{llll}
\hline \multirow{2}{*}{ Variables } & \multicolumn{2}{c}{ Univariate analysis } & \multicolumn{2}{c}{ Multivariate analysis } \\
\cline { 2 - 3 } Age at recurrence, y $(\leq 50$ vs. $>50)$ & $\mathrm{P}$ & $\mathrm{HR}$ & 1.238 \\
Tumor size, cm (1,2 vs. 3,4) & 0.4 & 0.732 \\
Number of LNM $(0,1$ vs. 2,3) & 0.42 & 0.657 & $0.042^{*}$ \\
First-line chemotherapy (CBC vs. PBC) & 0.5 & 0.536 & 0.514 \\
Tumor grade (II vs. III) & 0.05 & 0.756 & 0.733 \\
Prior anthracyclines (yes vs. no) & 0.38 & 0.593 & 0.056 \\
Prior taxanes (yes vs. no) & 0.46 & 0.537 & \\
Number of metastatic organs (single vs. multiple) & 0.21 & 0.065 & \\
\hline
\end{tabular}

*, P values <0.05. CBC, capecitabine-based chemotherapy; LNM, lymph node metastases; HR, hazard ratio; PBC, platinum-based chemotherapy.

Table 5 Percent frequency of selected treatment-related adverse events

\begin{tabular}{|c|c|c|c|c|c|c|}
\hline Toxicity & \multicolumn{2}{|c|}{ PBC (n=33), No. (\%) } & \multicolumn{2}{|c|}{ CBC (n=26), No. (\%) } & $\mathrm{P}$ (grade 1-2) & P (grade 3-4) \\
\hline Vomiting & $18(54.5)$ & $10(30.3)$ & $6(23.1)$ & $2(7.7)$ & $0.015^{*}$ & $0.045^{\star}$ \\
\hline Neutropenia & $25(75.8)$ & $7(21.2)$ & $10(38.5)$ & $4(15.4)$ & $0.004^{*}$ & 0.57 \\
\hline Hand-foot syndrome & 0 & 0 & $15(57.7)$ & $2(7.7)$ & $<0.001^{*}$ & $<0.001^{*}$ \\
\hline Fatigue & $11(33.3)$ & 0 & $9(34.6)$ & 0 & 0.92 & NA \\
\hline Hepatic abnormalities & $5(16.0)$ & $2(8.0)$ & $5(20.1)$ & $1(4.7)$ & 0.68 & 0.7 \\
\hline
\end{tabular}

*, indicates statistically significant. CBC, capecitabine-based chemotherapy; PBC, platinum-based chemotherapy; NA, not applicable. 
foot syndrome. In the $\mathrm{PBC}$ group, the incidence of gastrointestinal, leukopenia, and neutropenia adverse events was more common than that of CBC. These results are in line with the previous studies (30-33).

Although the treatment option for breast cancer LM is palliative, different local treatment modalities, such as surgery and stereotactic body radiotherapy (SBRT), have been applied together with systemic chemotherapeutic agents in order to improve outcomes $(34,35)$.

For breast cancer patients with LM receiving metastasectomy, the median 3-, and 5-year survival rates range between $49-94 \%$ and $5-78 \%$, respectively (36-39). Findings from a case-matched analysis showed that liver resection combined with systemic treatment resulted in improved OS compared to systemic treatment alone. Median OS of the resection group was 82 months with a 3 - and 5 -year OS of $81 \%$ and $69 \%$, respectively, compared with a median OS of 31 months in the systemic group with a 3 - and 5 -year OS of $32 \%$ and $24 \%$, respectively (40). A systematic review (41) analyzed 956 patients receiving resection of breast cancer LM. The median 3-, and 5-year survival were $52.9 \%$ and $33 \%$ respectively. Despite some promising reports, surgical resection of BCLM is still controversial because of its invasiveness. In addition, many patients develop unpredictable recurrent disease (42).

SBRT offers an alternative, non-invasive approach for LM, with highly conformal doses delivered to tumor sites and a steep dose gradient, which allows normal liver tissues to be spared. Retrospective and prospective studies have demonstrated the feasibility of SBRT for LM with local control rates ranging from $60-90 \%$ at 2 years after treatment $(43,44)$. Findings from a recent study revealed that SBRT might be an effective and safe treatment option in selected breast cancer patients with LM (45). Another study reported that the median OS after SBRT of breast patients with LM was 21 months (46). The patient selection criteria, and optimal dose and fractionation for liver SBRT are still under investigation. Several clinical trials evaluating SBRT on patients with limited MBC are on-going. An on-going randomized phase II/III trial (NCT02364557) studies how well standard of care therapy with stereotactic radiosurgery and/or surgery works and compares it to the standard of care therapy alone in treating patients with breast cancer that has spread to one or two locations in the body (limited metastatic) that are previously untreated. Another phase II/III multi-center randomized randomised controlled trial (The CORE study, NCT02759783) in patients with breast, prostate or non-small cell lung cancer
(NSCLC) primary cancer is comparing standard of care with or without SBRT for extra-cranial metastases, and will help to clarify whether SBRT is a viable therapeutic approach for breast cancer metastases.

Our study should be considered in the context of its limitations. First, this was a retrospective study and the sample size was relatively small. Second, TNBC is a heterogeneous disease and treatment response to chemotherapy might vary between basal and non-basal breast cancer. In spite of its limitations, this study provided clinical reference that capecitabine is considerably effective in TNBC with LM, and capecitabine, rather than platinum, might be a better choice in first-line chemotherapy for TNBC patients with $\mathrm{LM}$, if not contraindicated.

In conclusion, our study indicates that $\mathrm{CBC}$, with its acceptable toxicity profile, might be used as an effective alternative treatment in patients with TNBC LM. In the future, studies involving larger number of patients are needed, and more clinical trials could perhaps be carried out.

\section{Acknowledgments}

We thank our colleagues at the National Cancer Center/ Cancer Hospital, Chinese Academy of Medical Sciences and Peking Union Medical College, Beijing Chao-Yang Hospital, Chinese PLA General Hospital, and Beijing Sanhuan Cancer Hospital for their support and assistance. In addition, we would like to thank patients and their family members for supporting our study.

Funding: None.

\section{Footnote}

Reporting Checklist: The authors have completed the STROBE reporting checklist. Available at http://dx.doi. org/10.21037/atm-20-4590

Data Sharing Statement: Available at http://dx.doi. org/10.21037/atm-20-4590

Conflicts of Interest: All authors have completed the ICMJE uniform disclosure form (available at http://dx.doi. org/10.21037/atm-20-4590). The authors have no conflicts of interest to declare.

Etbical Statement: The authors are accountable for all aspects of the work in ensuring that questions related to the accuracy or integrity of any part of the work are 
appropriately investigated and resolved. The study was conducted in accordance with the Declaration of Helsinki (as revised in 2013). This study was approved by the Ethics Committee of National Cancer Center/National Clinical Research Center for Cancer/Cancer Hospital, Chinese Academy of Medical Sciences and Peking Union Medical College (No.: 15-115/1042). Because of the retrospective nature of the research, the requirement for informed consent was waived.

Open Access Statement: This is an Open Access article distributed in accordance with the Creative Commons Attribution-NonCommercial-NoDerivs 4.0 International License (CC BY-NC-ND 4.0), which permits the noncommercial replication and distribution of the article with the strict proviso that no changes or edits are made and the original work is properly cited (including links to both the formal publication through the relevant DOI and the license). See: https://creativecommons.org/licenses/by-nc-nd/4.0/.

\section{References}

1. Denkert C, Liedtke C, Tutt A, et al. Molecular alterations in triple-negative breast cancer-the road to new treatment strategies. Lancet 2017;389:2430-42.

2. Foulkes WD, Smith IE, Reis-Filho JS. Triple-negative breast cancer. N Engl J Med 2010;363:1938-48.

3. He ZY, Wu SG, Peng F, et al. Up-Regulation of RFC3 Promotes Triple Negative Breast Cancer Metastasis and is Associated with Poor Prognosis Via EMT. Transl Oncol 2017;10:1-9.

4. Adam R, Aloia T, Krissat J, et al. Is liver resection justified for patients with hepatic metastases from breast cancer? Ann Surg 2006;244:897-907.

5. Waks AG, Winer EP. Breast Cancer Treatment: A Review. JAMA 2019;321:288-300.

6. Estevez LG, Gradishar WJ. Evidence-based use of neoadjuvant taxane in operable and inoperable breast cancer. Clin Cancer Res 2004;10:3249-61.

7. Pacilio C, Morabito A, Nuzzo F, et al. Is epirubicin effective in first-line chemotherapy of metastatic breast cancer $(\mathrm{MBC})$ after an epirubicin-containing adjuvant treatment? A single centre phase III trial. Br J Cancer 2006;94:1233-6.

8. Fan Y, Xu BH, Yuan P, et al. Docetaxel-cisplatin might be superior to docetaxel-capecitabine in the first-line treatment of metastatic triple-negative breast cancer. Ann Oncol 2013;24:1219-25.
9. Tutt A, Tovey H, Cheang MCU, et al. Carboplatin in BRCA1/2-mutated and triple-negative breast cancer BRCAness subgroups: the TNT Trial. Nat Med 2018;24:628-37.

10. Hu XC, Zhang J, Xu BH, et al. Cisplatin plus gemcitabine versus paclitaxel plus gemcitabine as first-line therapy for metastatic triple-negative breast cancer (CBCSG006): a randomised, open-label, multicentre, phase 3 trial. Lancet Oncol 2015;16:436-46.

11. Barrett-Lee P, Bidard FC, Pierga JY. Contemporary issues and the potential uses of capecitabine in metastatic breast cancer. Cancer Treat Rev 2009;35:582-9.

12. Van Cutsem E, Twelves C, Cassidy J, et al. Oral capecitabine compared with intravenous fluorouracil plus leucovorin in patients with metastatic colorectal cancer: results of a large phase III study. J Clin Oncol 2001;19:4097-106.

13. Miwa M, Ura M, Nishida M, et al. Design of a novel oral fluoropyrimidine carbamate, capecitabine, which generates 5 -fluorouracil selectively in tumours by enzymes concentrated in human liver and cancer tissue. Eur J Cancer 1998;34:1274-81.

14. Shien T, Doihara H, Nishiyama K, et al. Clinical efficacy of capecitabine and cyclophosphamide (XC) in patients with metastatic breast cancer. Acta Med Okayama 2011;65:231-7.

15. Thomas ES, Gomez HL, Li RK, et al. Ixabepilone plus capecitabine for metastatic breast cancer progressing after anthracycline and taxane treatment. J Clin Oncol 2007;25:5210-17.

16. Miller KD, Chap LI, Holmes FA, et al. Randomized phase III trial of capecitabine compared with bevacizumab plus capecitabine in patients with previously treated metastatic breast cancer. J Clin Oncol 2005;23:792-9.

17. Fumoleau P, Largillier R, Clippe C, et al. Multicentre, phase II study evaluating capecitabine monotherapy in patients with anthracycline- and taxane-pretreated metastatic breast cancer. Eur J Cancer 2004;40:536-42.

18. Jiang $Y$, Yin $W$, Zhou L et al. First efficacy results of capecitabine with anthracycline- and taxane-based adjuvant therapy in high-risk early breast cancer: a meta-analysis. PLoS One 2012;7:e32474.

19. Xu D, Chen X, Li X, et al. Addition of Capecitabine in Breast Cancer First-line Chemotherapy Improves Survival of Breast Cancer Patients. J Cancer 2019;10:418-29.

20. Schuller J, Cassidy J, Dumont E, et al. Preferential activation of capecitabine in tumor following oral administration to colorectal cancer patients. Cancer 
Chemother Pharmacol 2000;45:291-7.

21. Miwa M, Ura M, Nishida M, et al. Design of a novel oral fluoropyrimidine carbamate, capecitabine, which generates 5 -fluorouracil selectively in tumours by enzymes concentrated in human liver and cancer tissue. Eur J Cancer 1998;34:1274-81.

22. Shien T, Doihara H, Nishiyama K, et al. Clinical efficacy of capecitabine and cyclophosphamide (XC) in patients with metastatic breast cancer. Acta Med Okayama 2011;65:231-7.

23. Malet-Martino M, Martino R. Clinical studies of three oral prodrugs of 5 - fluorouracil (capecitabine, UFT, S-1): a review. Oncologist 2002;7:288-323.

24. Miwa M, Ura M, Nishida M, et al. Design of a novel oral fluoropyrimidine carbamate, capecitabine, which generates 5-fluorouracil selectively in tumours by enzymes concentrated in human liver and cancer tissue. Eur J Cancer 1998;34:1274-81.

25. Nio Y, Kimura H, Tsubono M, et al. Antitumor activity of 50-deoxy-5-fluorouridine in human digestive organ cancer xenografts and pyrimidine nucleoside phosphorylase activity in normal and neoplastic tissues from human digestive organs. Anticancer Res 1992;12:1141-6.

26. Li XP, Meng ZQ, Guo WJ, et al. Treatment for liver metastases from breast cancer: results and prognostic factors. World J Gastroenterol 2005;11:3782-7.

27. Wang J, Xu B, Yuan P, et al. Capecitabine combined with docetaxel versus vinorelbine followed by capecitabine maintenance medication for first-line treatment of patients with advanced breast cancer: Phase 3 randomized trial. Cancer 2015;121:3412-21.

28. Estevez LG, Batista N, Sanchez-Rovira P, et al. A phase II study of capecitabine and vinorelbine in patients with metastatic breast cancer pretreated with anthracyclines and taxanes. Clin Breast Cancer 2008;8:149-54.

29. Lueck H, Minckwitz GV, Du Bois A, et al. Epirubicin/ paclitaxel (EP) vs. capecitabine/paclitaxel (XP) in first-line metastatic breast cancer (MBC): a prospective, randomized multicentre phase III study of the AGO Breast Cancer Study Group. J Clin Oncol 2006;24.

30. O'Shaughnessy J, Koeppen H, Xiao Y, et al. Patients with Slowly Proliferative Early Breast Cancer Have Low FiveYear Recurrence Rates in a Phase III Adjuvant Trial of Capecitabine. Clin Cancer Res 2015;21:4305-11.

31. Masuda N, Lee S J, Ohtani S, et al. Adjuvant Capecitabine for Breast Cancer after Preoperative Chemotherapy. N Engl J Med 2017;376:2147-59.

32. von Minckwitz G, Rezai M, Fasching PA, et al. Survival after adding capecitabine and trastuzumab to neoadjuvant anthracycline-taxane-based chemotherapy for primary breast cancer (GBG 40--GeparQuattro). Ann Oncol 2014;25:81-9.

33. von Minckwitz G, Möbus V, Schneeweiss A, et al. German adjuvant intergroup node-positive study: a phase III trial to compare oral ibandronate versus observation in patients with high-risk early breast cancer. J Clin Oncol 2013;31:3531-9.

34. Caivano D, Valeriani M, Russo I, et al. Stereotactic body radiation therapy in primary and metastatic liver disease. Anticancer Res 2017;37:7005.e10.

35. Goodman BD, Mannina EM, Althouse SK, et al. Longterm safety and efficacy of stereotactic body radiation therapy for hepatic oligometastases. Pract Radiat Oncol 2016;6:86.e95.

36. Ruiz A, van Hillegersberg R, Siesling S, et al. Surgical resection versus systemic therapy for breast cancer liver metastases: Results of a European case matched comparison. Eur J Cancer 2018;95:1-10.

37. He X, Zhang Q, Feng Y, et al. Resection of liver metastases from breast cancer: a multicentre analysis. Clin Transl Oncol 2020;22:512-21.

38. Ercolani G, Zanello M, Serenari M, et al. Ten-Year Survival after Liver Resection for Breast Metastases: A Single-Center Experience. Dig Surg 2018;35:372-80.

39. Bacalbasa N, Balescu I, Ilie V, et al. The Impact on the Long-term Outcomes of Hormonal Status After Hepatic Resection for Breast Cancer Liver Metastases. In Vivo 2018;32:1247-53.

40. Ruiz A, van Hillegersberg R, Siesling S, et al. Surgical resection versus systemic therapy for breast cancer liver metastases: Results of a European case matched comparison. Eur J Cancer 2018;95:1-10.

41. Fairhurst K, Leopardi L, Satyadas T, et al. The safety and effectiveness of liver resection for breast cancer liver metastases: A systematic review. Breast 2016;30:175-84.

42. Fairhurst K, Leopardi L, Satyadas T, et al. The safety and effectiveness of liver resection for breast cancer liver metastases: A systematic review. Breast 2016;30:175-84.

43. Lee MT, Kim JJ, Dinniwell R, et al. Phase I study of individualized stereotactic body radiotherapy of liver metastases. J Clin Oncol 2009;27:1585-91.

44. Scorsetti M, Clerici E, Comito T. Stereotactic body radiation therapy for liver metastases. J Gastrointest Oncol 2014;5:190-7.

45. Onal C, Guler OC, Yildirim BA. Treatment outcomes of breast cancer liver metastasis treated with stereotactic body 
radiotherapy. Breast 2018;42:150-6.

46. Mahadevan A, Blanck O, Lanciano R, et al. Stereotactic Body Radiotherapy (SBRT) for liver metastasis - clinical outcomes from the international multi-institutional RSSearch® Patient Registry. Radiat Oncol 2018;13:26.

Cite this article as: Chen Y, Guan Y, Wang J, Ma F, Luo Y, Chen S, Zhang P, Li Q, Cai R, Li Q, Mo H, Fan Y, Zhao W, Xu B. Comparison of capecitabine-based regimens with platinumbased regimens in Chinese triple-negative breast cancer patients with liver metastasis. Ann Transl Med 2021;9(2):109. doi: 10.21037/atm-20-4590 